www.jmscr.igmpublication.org

Impact Factor (SJIF): 6.379

Index Copernicus Value: 79.54

ISSN (e)-2347-176x ISSN (p) 2455-0450

crossrefDOI: https://dx.doi.org/10.18535/jmscr/v6i8.153

Journal Of Medical Science And Clinical Research

\title{
Bladder Tumor in Young Boy: A Rare Case Report
}

\author{
Authors \\ Dr Sumit Gupta ${ }^{1}$, Dr Piyush Aggarwal ${ }^{2}$, Dr Kh Somarendra ${ }^{3}$ \\ ${ }^{1}$ Mch Urology Student, ${ }^{2}$ M.S General Surgery, ${ }^{3}$ Assistant Professor Urology
}

\begin{abstract}
Introduction: Bladder cancer below the age of 20 years old is not only rare, with an approximated total of 125 cases being collected worldwide by 2010 but it has also been defined as clinico-pathologically distinct from bladder tumors in older age groups. As benign causes of frank hematuria are more common in this age group than tumors, this often leads to a considerable delay in the diagnosis. With this case we would like to emphasize that bladder tumor should be consider in the diagnosis for evaluation of causes for hematuria in younger age groups.

Case Report: We present a case of 15 year old boy who came to us with on and off hematuria since 4 months with occasional passage of clots. No other significant history. No family history of malignancy. No history of smoking. On examination- he had pallor, dehydration and suprapubic tenderness. He was admitted and received 4 units of PRBC for hemodynamic stabilization.

A microscopy and culture test of his urine confirmed his urine to be sterile and urine cytology was negative for malignant cells. On further workup USG showed $5.2 \times 3 \mathrm{~cm}$ Urinary Bladder growth in right posteroinferior wall suggesting urinary bladder tumor. CECT revealed small polypoidal irregular soft tissue mass in posterolateral wall of urinary bladder and no evidence of any upper tract abnormality, lymph nodes or metastases. He underwent TURBT under spinal anesthesia and perioperative periods were uneventful. HPE reported as low grade papillary urothelial cancer. He was discharged with follow up advice.

Conclusion: Despite its low incidence in children, TCCB must be suspected in the event of macroscopic haematuria. Ultrasound followed by cystoscopy are the ideal diagnostic tools for visualization of these tumours. Follow up must be clinical with periodic ultrasound evaluation. Periodic cystoscopy is indicated only in cases of clinical or ultrasonographic suspicion of recurrence.

Discussion: Neither physical examination nor laboratory analysis revealed any significant abnormalities, but ultrasound showed polypoidal intravesical lesions. Surgical resection was performed endoscopically.
\end{abstract}

\section{Introduction}

Bladder cancer below the age of 20 years old is not only rare, with an approximated total of 125 cases being collected worldwide by $2010{ }^{1}$ but it has also been defined as clinico-pathologically distinct from bladder tumours in older age groups ${ }^{2}$. The majorities is low grade superficial transitional cell carcinomas and are associated with a low rate of recurrence ${ }^{3}$.
As benign causes of frank haematuria are more common in this age group than tumours, this often leads to a considerable delay in the diagnosis ${ }^{2}$. According to the literature, there are time delays of up to 1 year or more from initial onset of symptoms to diagnosis, in more than a quarter of cases ${ }^{2}$. It is understandable that clinicians have a low index of suspicion in this group of patients when a study like that conducted by Greenfield et 
al., showed that only 3 children/young adults (all aged less than 20) out of 342 who presented with frank haematuria were eventually diagnosed with bladder tumours ${ }^{4}$. There is also less inclination to expose young people to a general anaesthesia to perform a cystoscopy and possibly causing urethral trauma. However, this important diagnosis should not be missed or delayed and clinicians' should have high index of suspicion for bladder cancer in the under age 20 group who present with haematuria to achieve the best prognostic outcomes.

In adults, management of bladder carcinoma involves transurethral resection for non-invasive lesions, and a cystectomy in cases of muscle invasion. In addition, surgery is typically followed by intravesical chemotherapy and/or immunotherapy in order to control any microscopic residuals ${ }^{5}$. However, such recommendations do not seem justified for children, who appear to have clinical features that differ from adult patients and a better prognosis ${ }^{6}$.

\section{Case Report}

We present a case of 15 year old boy who came to us with on and off hematuria since 4 months with occasional passage of clots. No other significant history. No family history of malignancy. No history of smoking. On examination- he had pallor, dehydration and suprapubic tenderness. He was admitted and received 4 units of PRBC for hemodynamic stabilization. A microscopy and culture test of his urine confirmed his urine to be sterile and urine cytology was negative for malignant cells. On further workup USG showed $5.2 \times 3 \mathrm{~cm}$ bladder growth in right postero-inferior wall suggesting urinary bladder tumor. Contrast Enhanced CT of whole abdomen revealed $3 \mathrm{~cm}$ polypoidal mass in right posterolateral wall of urinary bladder with no evidence of any upper tract changes, enlarged lymph nodes or metastases (fig 1). He underwent TURBT under spinal anesthesia (fig 2) with complete resection of tumor. perioperative period was uneventful. HPE reported as low grade non muscle invasive papillary urothelial cancer (fig 3). He was discharged with follow up advice. Patient was followed for 1 year postoperatively with no evidence of recurrence.

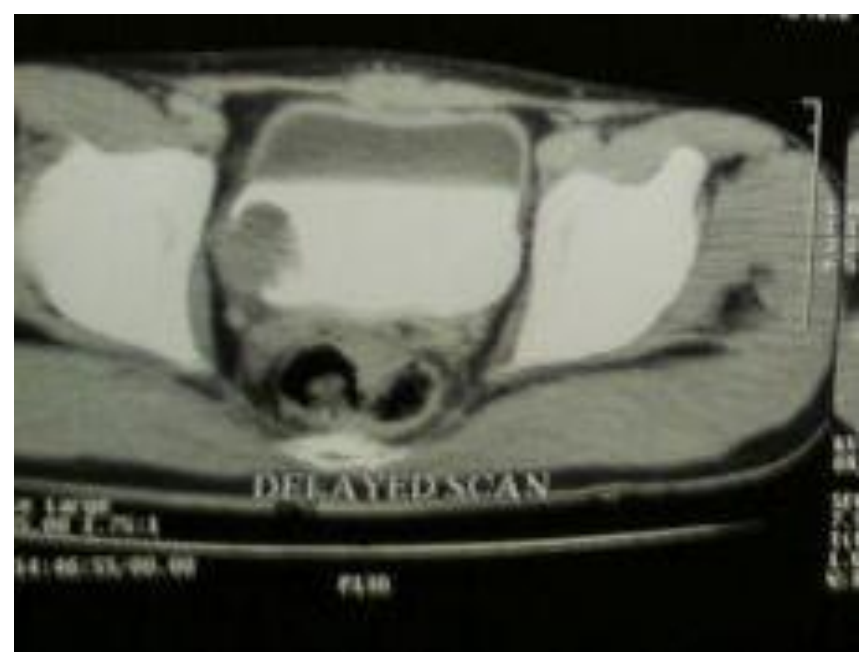

Fig 1

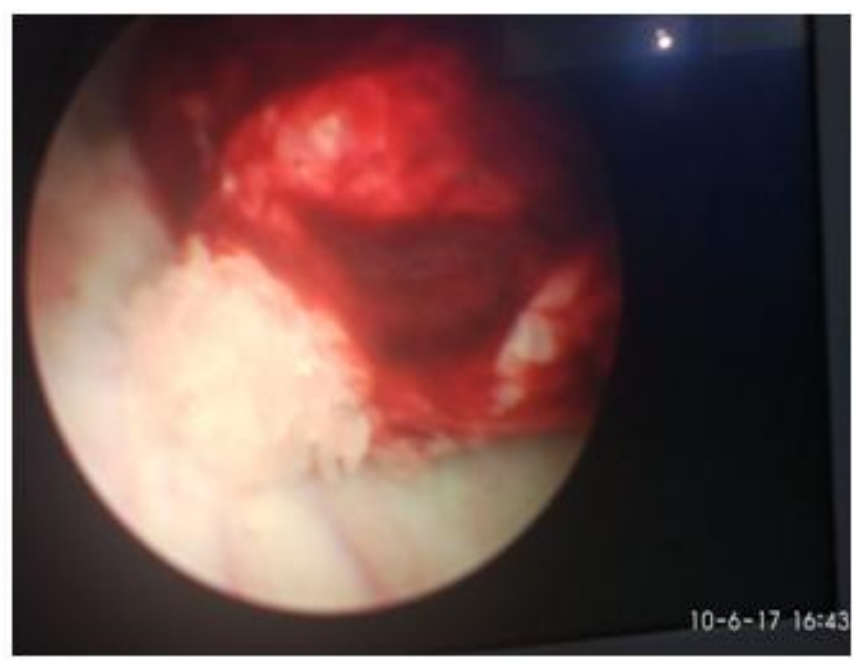

Fig 2

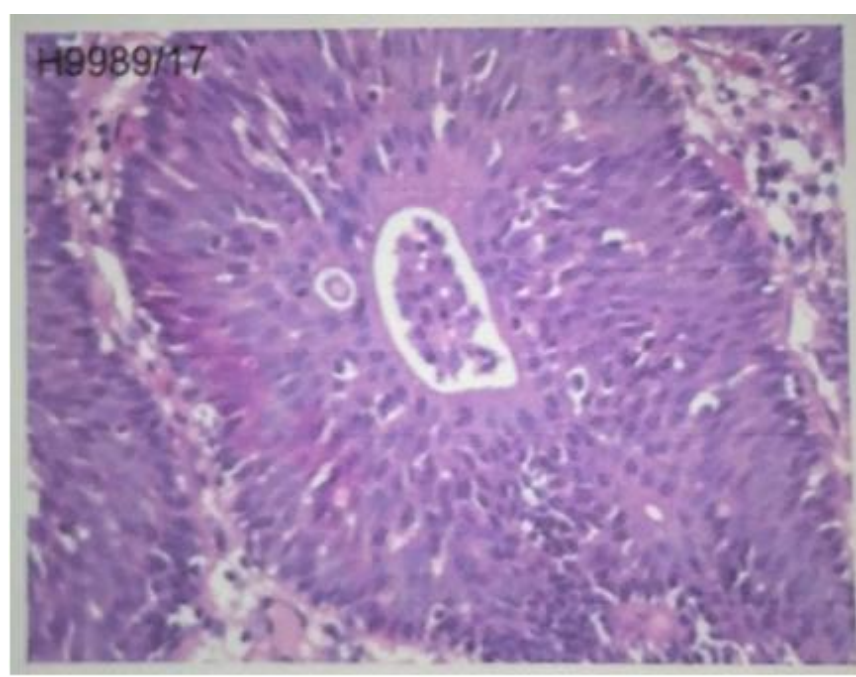

Fig 3 


\section{Discussion}

Urothelial carcinoma of the bladder in the first two decades of life is extremely rare. This becomes more true in the case of muscle invasive. Previous studies reported bladder cancer to be superficial in pediatric and young adult patients ${ }^{7}$. Paner et al. in a comprehensive review of bladder cancer in patients younger than 30 years of age, only $3.0 \%$ had muscle-invasive disease and only $1.7 \%$ had high-grade tumor $^{8}$. Since the vast majority of young patients with bladder tumor present with non-muscle invasive and low-grade disease, these patients also have lower progression and recurrence rates compared to older patients. This further suggested that its nature in younger population is different compared to elderly patients. On the other hand, Yossepowitch and Dalbagni found no difference in grade or stage upon comparing 74 patients $<40$ years of age to 75 patients $>65$ years of age ${ }^{9}$. However, when focusing on only bladder cancer patients diagnosed in the first two decades of life, it is evident that these patients had a relatively indolent be having bladder cancer ${ }^{8}$. However, aggressive bladder cancer has been reported in children - a 31 month old ${ }^{10}$ anda 14 year old ${ }^{11}$. Risk factors for bladder cancer in young patients are not clear. Cigarette smoking is by far the most prevalent risk factor for bladder cancer. Exposure to polycyclic aromatic hydrocarbons (PAHs) is responsible for $10 \%$ to $15 \%$ of cases as a result of bladder carcinogenesis. Genetic predisposition to bladder cancer has been suggested by few previous reports in families with bladder cancer. However, it is unclear whether it resulted from a genetic predisposition or common environmental exposure among family members ${ }^{12}$.Gross hematuria is the most common symptom of bladder tumor in both pediatric as well as in adult patients. Hematuria often misleads to diagnosis of urinary tract infection or urinary stone disease $\mathrm{e}^{13,14}$ and thus, the underlying condition remains unidentified.

\section{Conclusions}

In conclusion, it is important to consider carefully presentations of gross hematuria in pediatric patients. In general, young patients with bladder tumor tend to have a favorable outcome. This tendency is more common in patients presenting early in life and is likely to decrease with age. CT scan followed by cystoscopy is the ideal diagnostic tools for visualization of these tumours.

\section{References}

1. Lerena J., Krauel L., García-Aparicio L., Vallasciani S., Suñol M., Rodó J. Transitional cell carcinoma of the bladder in children and adolescents: six-case series and review of the literature. J. Pediatr Urol. 2010;6:481-485.

2. Paner G.P., Zehnder P., Amin A.M. Urothelial neoplasms of the urinary bladder occurring in young adult and pediatric patients: a comprehensive review of literature with implications for patient management. Adv. Anat. Pathol. 2011; 18:79-89.

3. Alanee S., Shukla A.R. Bladder malignancies in children aged $<18$ years: results from the Surveillance, Epidemiology and End Results database. BJU Int. 2010;106:557-560.

4. Greenfield S.P., Williot P., Kaplan D. Gross hematuria in children: a ten-year review. Urology. 2007;69:166-169.

5. Resnick MJ, Bassett JC, Clark PE. Management of superficial and muscleinvasive urothelial cancers of the bladder. Curr OpinOncol. 2013;25:281-288.

6. Yusim I, Lismer L, Greenberg G, Haomud $\mathrm{K}$, Kaneti J. Carcinoma of the bladder in patients under 25 years of age. Scand J Urol Nephrol. 1996;30:461-463. doi: 10.3109/00365599609182324.

7. Fine SW, Humphrey PA, Dehner LP, Amin MB, Epstein JL. Urothelial neoplasms in patients 20 years or younger: a clinicopathological analysis using the 
world health organization 2004 bladder consensus classification. J Urol. 2005;174: 1976-1980.

8. Paner GP, Zehnder P, Amin AM, Husain AN, Desai MM. Urothelial neoplasms of the urinarybladder occurring in young adult and pediatric patients: a comprehensive review of literature with implications for patient management. Adv Anat Pathol. 2011;18:79-89.

9. Yossepowitch O, Dalbagni G. Transitional cell carcinoma of the bladder in young adults: presentation, natural history and outcome. J Urol. 2002;168:61-66.

10. Lezama-del Valle P, Jerkins GR, Rao BN, Santana VM, Fuller C, Merchant TE. Aggressive bladder carcinoma in a child. Pediatr Blood Cancer. 2004;43:285-288.

11. Scott AA, Stanley W, Worsham GF, Kirkland TA, Jr, Gansler T, Garvin AJ. Aggressive bladder carcinoma in an adolescent. Report of a case with immunohistochemical, cytogenetic, and flow cytometric characterization. Am J Surg Pathol. 1989;13:1057-1063.

12. Mueller CM, Caporaso N, Greene MH. Familial and genetic risk of transitional cell carcinoma of the urinary tract. Urol Oncol. 2008;26:451-464.

13. Ristic-Petrovic A, Stojnev S, JankovićVelickoviċ L, Marjanovic G. Malakoplakia mimics urinarybladder cancer: A case report. Vojnosanit Pregl. 2013;70:606608.

14. Gedikoğlu G, Sökmensüer C, Söylemezoğlu F, Koçal C. Carcinosarcoma of the urinary bladder. IntUrolNephrol. 1996;28:333-336. 